\title{
Performance Analysis of 3D Massive MIMO Cellular Systems with Collaborative Base Station
}

\author{
Xingwang Li, Lihua Li, Ling Xie, Xin Su, and Ping Zhang \\ State Key Laboratory of Networking and Switching Technology, Beijing University of Posts and Telecommunications, \\ Beijing 100786, China \\ Correspondence should be addressed to Lihua Li; lilihua@bupt.edu.cn
}

Received 24 January 2014; Revised 16 May 2014; Accepted 24 June 2014; Published 10 July 2014

Academic Editor: Xuefeng Yin

Copyright (C) 2014 Xingwang Li et al. This is an open access article distributed under the Creative Commons Attribution License, which permits unrestricted use, distribution, and reproduction in any medium, provided the original work is properly cited.

\begin{abstract}
Massive MIMO have drawn considerable attention as they enable significant capacity and coverage improvement in wireless cellular network. However, pilot contamination is a great challenge in massive MIMO systems. Under this circumstance, cooperation and three-dimensional (3D) MIMO are emerging technologies to eliminate the pilot contamination and to enhance the performance relative to the traditional interference-limited implementations. Motivated by this, we investigate the achievable sum rate performance of MIMO systems in the uplink employing cooperative base station (BS) and 3D MIMO systems. In our model, we consider the effects of both large-scale and small-scale fading, as well as the spatial correlation and indoor-to-outdoor high-rise propagation environment. In particular, we investigate the cooperative communication model based on 3D MIMO and propose a closed-form lower bound on the sum rate. Utilizing this bound, we pursue a "large-system" analysis and provide the asymptotic expression when the number of antennas at the BS grows large, and when the numbers of antennas at transceiver grow large with a fixed ratio. We demonstrate that the lower bound is very tight and becomes exact in the massive MIMO system limits. Finally, under the sum rate maximization condition, we derive the optimal number of UTs to be served.
\end{abstract}

\section{Introduction}

Multiple-input multiple-output (MIMO) technology can provide a remarkable increase in data rate and reliability compared to the single-antenna system $[1,2]$, without sacrificing extra bandwidth or transmit power. Recently, a novel network architecture referred to as massive MIMO or large-scale MIMO has been proposed with significant potential of both increasing the spectral efficiency and providing significant power saving $[3,4]$. And it has a disruptive potential for the future $5 \mathrm{G}$ wireless communication [5]. However, deploying a huge number of antennas in either transmitters or receivers is a great challenge. Moreover, pilot contamination is branded as a fundamental limit for massive MIMO systems [6]. Therefore, three-dimensional (3D) MIMO [7-10] and cooperation [11-14] are introduced as the promising techniques in massive MIMO network to enhance the performance of cell-edge user terminals (TUs) and eliminate the pilot contamination.

Currently, most of researches on MIMO systems are based on traditional two-dimensional (2D) MIMO channel model, which only involves the horizontal dimension while ignoring the effect of tilt angle in the vertical dimension. However, antenna tilt angle has a large impact on the performance of cooperative communication system as shown in [15]. In this case, 3D MIMO is a promising technology to improve the throughput of the cell-edge UTs and the degree of fairness between the cell-edge UTs and cell-center UTs [16]. The basic idea of 3D MIMO is to serve UTs close to the base station (BS) with large tilt angles and to serve UTs at the cell boundary with smaller ones. Nevertheless, a disadvantage of 3D MIMO is the potential of intercell interference aggravation caused by the UTs close to the cell boundary [9]. To overcome this issue, cooperative techniques, such as BS cooperation, relaying, and UT conferencing are often considered as effective methods to cope with the intercell interference.

BS cooperation is often referred to as multicell processing in which the BSs jointly decode the message based on the received signals (also known as coordinated multipoint (CoMP)). The capacity enhancement due to the BS 
cooperation has been extensively studied and has been shown to grow linearly with the number of $\mathrm{BS}$ receive antennas [17, 18]. However, the common characteristic of $[17,18]$ is that they consider the simplified assumption that the fading coefficients of the MIMO subchannels are completely uncorrelated. In $[19,20]$, using tools of random matrix theory (RMT), the authors derive a deterministic approximation of uplink sum rate capacity with minimum mean-square error (MMSE) receiving. However, since the limit of the sum rate obtained therein is deterministic, the final expression does not enable further manipulations. More importantly, 3D MIMO and large-scale fading, as well as the correlation at the transmit side and indoor-to-outdoor (I2O) high-rise wireless propagation environments, are not taken into account.

In this paper, we focus on the cooperating BSs which are interconnected through ideal links (e.g., optical fiber or high speed cable) to a central processor (CP), which has perfect channel state information (CSI). In detail, we investigate the sum rate performance of the 3D massive MIMO systems over BS cooperation in composite fading channels considering the effect of spatial correlation at the transmit side and I2O wireless propagation environment. In this scenario, we assume that the cell-edge UTs are uniformly distributed in a building with several floors and cell-center UTs are uniformly distributed in other areas of the cell. We note that the spatial nonstationary property and the near-field effect are the main properties for the realistic massive MIMO channel model which are interesting topics for additional research [21, 22]. In this paper, the analysis of the effects of the two properties is not taken into account due to the space constraints, which is left to the future work. The contributions of this paper can be summarized as follows.

(1) A cellular 3D MIMO uplink channel model is introduced, accommodating $\mathrm{I} 2 \mathrm{O}$ high-rise propagation environments, Rayleigh lognormal fading model, distance dependent loss, and Kronecker correlated antennas.

(2) We derive a closed-form lower bound on the sum rate performance of the $3 \mathrm{D}$ MIMO ZF receivers. This bound does not involve complicated functions, and it can be computed fast and efficiently.

(3) With the help of the proposed lower bound, we analyze the asymptotic lower bound for 3D massive MIMO system under the cases that the average and total powers are fixed, respectively. The proposed bound applies to any finite number of antennas and remains relatively tight across the entire SNR and tilt angle ranges.

(4) Exploiting the results of (2), we also give a closedform approximated solution for the number of UTs, $K$, which maximizes the sum rate under a fixed receive antenna number.

The rest of this paper is organized as follows. Section 2 outlines the multicell processing of uplink 3D MIMO fading channel models. In Section 3, we provide a novel analytical lower bound on the sum rate performance of 3D MIMO system in which the BSs exploit variable elevation, while
Section 4 details about the sum rate and the asymptotic analysis for 3D massive MIMO. The analysis of the UT number which optimizes the performance of the sum rate is also investigated. We present some numerical results and the corresponding analysis in Section 5 before we conclude the paper in Section 6.

\section{Systems Model}

2.1. MIMO Channel Fading Model. In the following, we consider $L$ cells with BS cooperation in the uplink of $3 \mathrm{D}$ MIMO cellular network. Each cell is partitioned into 3 sectors with $K$ UTs served within the coverage of each sector. Each BS corresponding to one sector is equipped with $N_{r}$ receive antennas, while each UT has $N_{t}$ transmit antennas $\left(N_{r} \geq\right.$ $\left.K N_{t}\right)$. Let $K_{e}$ and $K_{c}$ represent the number of UTs which are uniformly located in the cell-edge located $F$ floor building and in other cell-center area, respectively, where $K$ satisfies $K=K_{e}+K_{c}$. A schematic illustration of the cellular system under consideration is depicted in Figure 1. Assuming that the channel state information (CSI) is unknown at the transmitters but known at all receivers to perform the zeroforcing $(\mathrm{ZF})$ detecting, then the available average power, $P$, is identical amongst all UTs. Under the conditions of flat fading, the received signal at the $l$ th cell is given by

$$
\begin{gathered}
\mathbf{y}_{l}=\sum_{i=1}^{L} \underbrace{\left(\sum_{k=1}^{k_{e}} \sqrt{a_{l i k} \varphi_{l i k} \xi_{l i k}} \mathbf{v}_{l i k}\left(\mathbf{R}_{l i k}\right)^{1 / 2} \mathbf{x}_{l i k}\right.}_{\text {cell-edge }} \\
+\underbrace{\left.\sum_{k=k_{e}+1}^{K} \sqrt{a_{l i k} \varphi_{l i k} \xi_{l i k}} \mathbf{v}_{l i k}\left(\mathbf{R}_{l i k}\right)^{1 / 2} \mathbf{x}_{l i k}\right)}_{\text {cell-center }}+\mathbf{z}_{l},
\end{gathered}
$$

where $\mathbf{x}_{l i k}$ is the vector of the transmitted signal by the $k$ th UT of the $i$ th cell $k=1, \ldots, K, i=1, \ldots, L, l=$ $1, \ldots, L, \mathbf{v}_{\text {lik }}$ is a $N_{r} \times N_{t}$ random matrix representing the corresponding small scale fading channel matrix, and the entries of the matrix are modeled as independent identically distributed (i.i.d.) complex Gaussian random variables (RVs) with zero mean and unit variance, while $\mathbf{R}_{l i k}$ is a $N_{t} \times N_{t}$ positive definite covariance matrix. The vector $\mathbf{z}_{l}$ represents the additive white Gaussian noise (AWGN) with zero mean, $E\left[\mathbf{z}_{l} \mathbf{z}_{l}^{H}\right]=N_{0} \mathbf{I}$. Note that the small scale fading correlation occurs only between the antennas of the same UT since the UTs are, in general, geographically separated. The coefficient $a_{l i k}$ is the $3 \mathrm{D}$ antenna gain between the $l$ th $\mathrm{BS}$ and the $k$ th UT in the $i$ th cell. The path loss $\varphi_{\text {lik }}$ includes the I2O and outdoor path loss models which are calculated according to the simplified 3GPP standard model in [23] and the modified path loss model in [19] due to small distance between the BS and UTs, respectively,

$$
\varphi_{l i k}= \begin{cases}\left(1+d_{l i k}\right)^{-v} \varphi_{l i k}^{\mathrm{tw}} \varphi_{l i k}^{\mathrm{in}}, & 1 \leq k \leq k_{e}, \\ \left(1+d_{l i k}\right)^{-v}, & k_{e}<k \leq K,\end{cases}
$$

where $d_{l i k}$ (all " $d$-values" in meter) is the distance between the $l$ th receiver and the $k$ th UT of the $i$ th cell while $v$ is the 


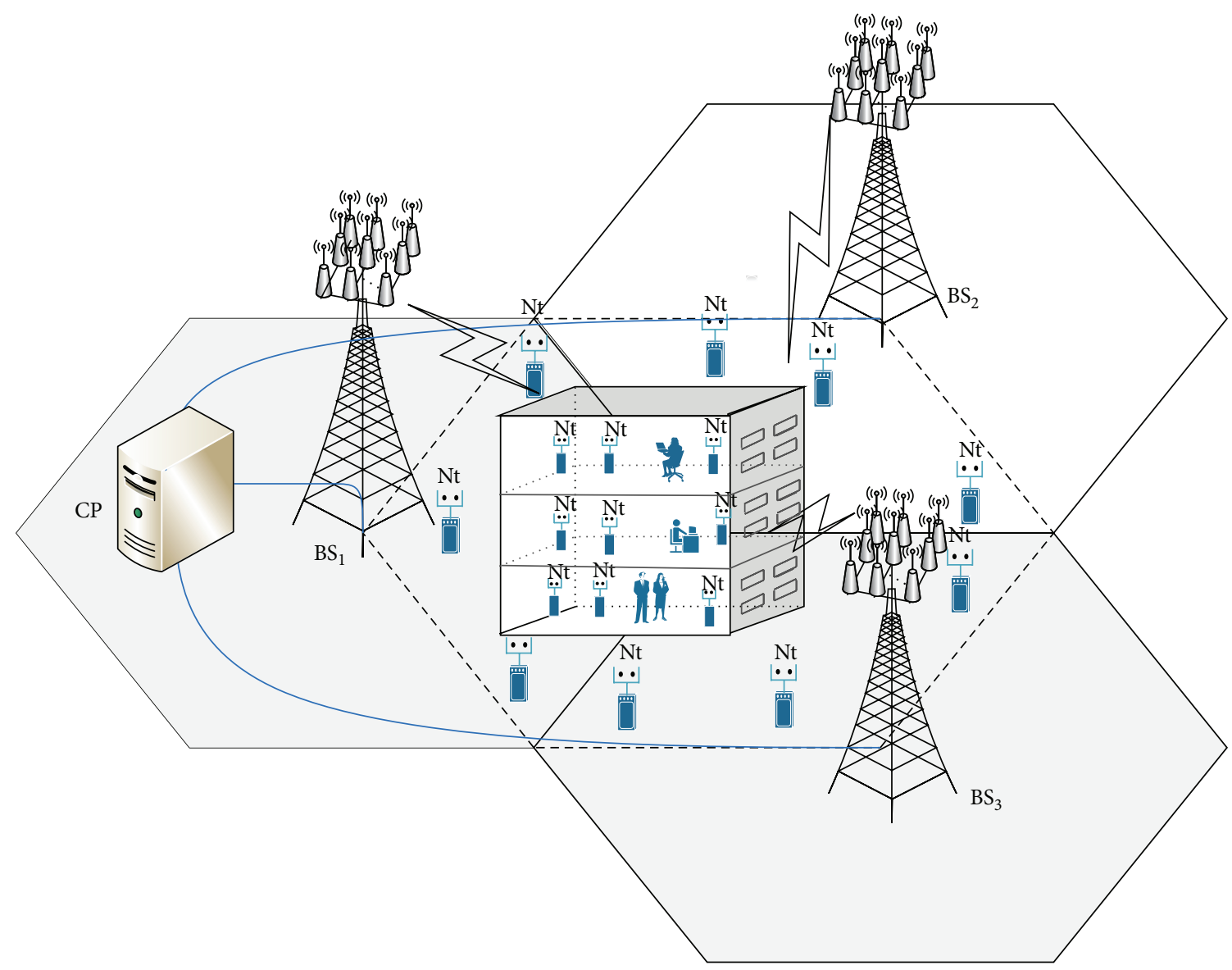

FIGURE 1: System model of coordinated multicell 3D MIMO network $(L=3)$.

path-loss exponent. $\varphi_{l i k}^{\mathrm{tw}}$ and $\varphi_{l i k}^{\mathrm{in}}$ are the wall penetration loss and the indoor propagation loss, respectively, whose values are determined by [23]. The lognormal fading model is the well-known shadowing model which is a prevalent model in the characterization of the shadowing fading MIMO channels in various environments [24-26]. In this case, the probability density function (PDF) of the shadowing fading coefficients $\xi_{l i k}$ is given by

$$
\begin{gathered}
p\left(\xi_{l i k}\right)=\frac{\eta}{\sqrt{2 \pi \sigma} \xi_{l i k}} \exp \left[-\frac{\left(10 \log _{10}\left(\xi_{l i k}\right)-\mu_{l i k}\right)^{2}}{2\left(\sigma_{l i k}\right)^{2}}\right], \\
\xi_{l i k} \geq 0,
\end{gathered}
$$

where $\eta=10 / \ln 10=4.3429, \mu_{l i k}(\mathrm{~dB})$, and $\sigma_{\text {lik }}(\mathrm{dB})$ are the mean and the standard deviation of $10 \log _{10}\left(\xi_{l i k}\right)$, respectively.

Note that all UTs are classified into two categories: (i) celledge UTs, of which the index value is smaller than or equal to $K_{e}$, are distributed in the building and (ii) cell-center UTs, of which the index value is larger than $K_{e}$ and smaller than or equal to $K$, are located distributing in the other area of the cell.

2.2. 3D MIMO Model. The radiation pattern for the $3 \mathrm{D}$ antenna elements of the BS array follows the model proposed

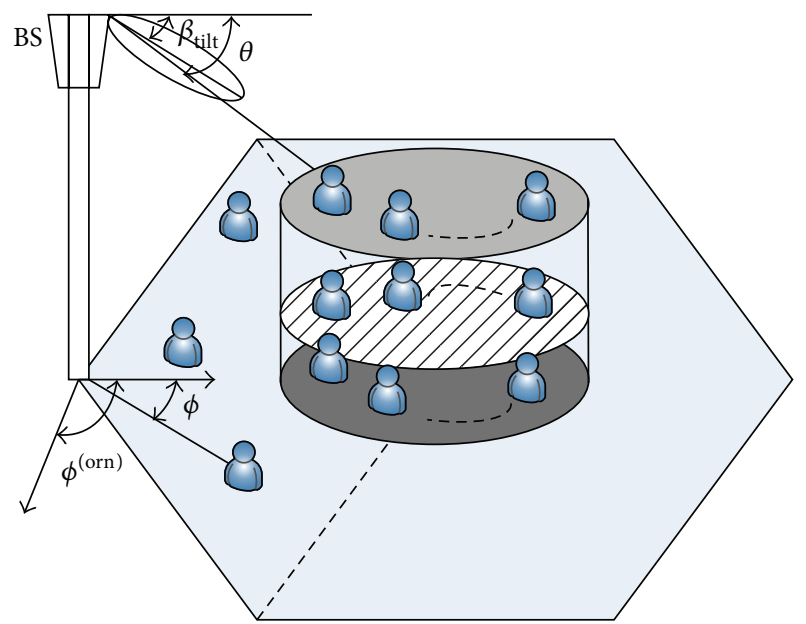

FIGURE 2: Schematic illustration of 3D MIMO in single cell.

by 3GPP in [23]. The 3GPP antenna pattern is a simple model which can accurately predict the signal propagation [27]. In this paper, we use the typical 3D radiation pattern (all " $g$ values" in decibel) as illustrated in Figure 2. In the following, 
we give the antenna gains for all UTs. The horizontal radiation pattern is given as

$$
g_{l i k}^{(H)}\left(\phi_{l i k}\right)=-\min \left[12\left(\frac{\phi_{l i k}-\phi_{l}^{(\text {orn })}}{\phi_{3 \mathrm{~dB}}}\right)^{2}, A_{m}\right]
$$

and the vertical radiation pattern is given by

$$
g_{l i k}^{(V)}(\theta)=-\min \left[12\left(\frac{\theta_{l i k}-\beta_{l}^{e}-\beta_{l}^{m}}{\theta_{3 \mathrm{~dB}}}\right)^{2}, \mathrm{SLA}_{v}\right],
$$

where $\phi_{l i k}$ is the azimuth angle between the $k$ th UT in the $i$ th cell and the horizontal main lobe of the $l$ th BS array radiation boresight, $-180^{\circ} \leq \phi_{l i k} \leq 180^{\circ} ; \phi_{l}^{(\text {orn })}$ is the fixed orientation angle of the $l$ th $\mathrm{BS}$ array radiation boresight; $\phi_{3 \mathrm{~dB}}$ is the horizontal half-power beam-width (HPBW); $A_{m}$ is the azimuth front-to-back ratio attenuation; $\theta_{l i k}$ is the elevation angle between the $k$ th UT in the $i$ th cell and the elevation main lobe of the $l$ th BS array radiation boresight; $\beta_{l}^{e}$ and $\beta_{l}^{m}$ are the electrical tilt angle and the mechanical tilt angle of the $l$ th BS which are both positive when tilting below the horizontal plane; $\theta_{3 \mathrm{~dB}}$ is the vertical HPBW; $\mathrm{SLA}_{v}$ is the side lobe attenuation. The composite $3 \mathrm{D}$ antenna pattern is obtained as

$$
g_{l i k}(\phi, \theta)=-\min \left\{-\left[g_{l i k}^{(H)}(\phi)+g_{l i k}^{(V)}(\theta)\right], A_{m}\right\} .
$$

Further, we can obtain the composite antenna pattern in numerical value as follows:

$$
a_{l i k}(\phi, \theta)=10^{g_{i k}(\phi, \theta) / 10} .
$$

It is noteworthy that adjusting antenna mechanical tilt angle requires a site visiting, which makes the adjustment process more expensive and time consuming. Therefore, all works in this paper assume a fixed mechanical tilt angle and only the electrical tilt angular variation is investigated.

2.3. Cellular Uplink Model. Under the condition of BS cooperation, the aforementioned model in Section 2.1 can be more compactly expressed as a vector memory-less channel with the form according to [19]

$$
\mathbf{Y}=\sqrt{\frac{P}{L K N_{t}}} \mathbf{G X}+\mathbf{Z},
$$

where $\mathbf{Y}=\left[\mathbf{y}_{1} \cdots \mathbf{y}_{L}\right]^{\mathrm{T}} \in \mathbb{C}^{L N_{r} \times 1}$ with $\mathbf{y}_{i}=\left[y_{i 1} \cdots y_{i N_{r}}\right] \epsilon$ $\mathbb{C}^{N_{r} \times 1}$ representing the received signal vector by the $N_{r}$ antennas of the $i$ th BS. For $\mathbf{X}=\left[\mathbf{X}_{1} \cdots \mathbf{X}_{L}\right]^{\mathrm{T}}$ we have $\mathbf{X}_{i}=\left[\mathbf{x}_{i 1} \cdots \mathbf{x}_{i K}\right]^{\mathrm{T}}$ and $\mathbf{x}_{i k}=\left[x_{i k 1} \cdots x_{i k N_{t}}\right] \in \mathbb{C}^{N_{t} \times 1}$ and $\mathbf{x}_{i k}$ representing the transmit signal vectors by the $k$ th UT of the $i$ th cell. The AWGN noise $\mathbf{Z}=\left[\mathbf{z}_{1} \cdots \mathbf{z}_{L}\right]^{\mathrm{T}}$ with $\mathbf{z}_{i}=\left[z_{i, 1} \cdots z_{i, N_{r}}\right] \in \mathbb{C}^{N_{r} \times 1}$ being i.i.d complex circularly symmetric (c.c.s) RVs.

The channel matrix $\mathbf{G} \in \mathbb{C}^{L N_{r} \times L K N_{t}}$ models the smallscale and large-scale fading, which can be given as follows:

$$
\mathbf{G}=\mathbf{H} \mathbf{\Omega}^{1 / 2},
$$

where $\mathbf{H} \in \mathbb{C}^{L N_{r} \times L K N_{t}}$ denotes the small-scale fading. The small-scale is assumed to follow a complex zero-mean Gaussian distribution with correlation between each row. Hence, we have

$$
\mathbf{H}=\mathbf{V R}^{1 / 2}=\mathbf{V}\left(\bigoplus_{l=1}^{L}\left(\bigoplus_{k=1}^{K} \mathbf{R}_{l l k}\right)\right)^{1 / 2},
$$

where the matrix $\mathbf{V} \in \mathbb{C}^{L N_{r} \times L K N_{t}}$ is the standard complex Gaussian matrix with the entries of zero-mean and unit variance while the matrix $\mathbf{R}$ is the transmit positive-define correlated covariance matrix. Here $\oplus$ represents the direct sum of matrices.

The elements of the diagonal matrix $\boldsymbol{\Omega}$ denote the largescale fading coefficients which can be expressed by

$$
\begin{aligned}
\boldsymbol{\Omega} & =\bigoplus_{l=1}^{L}\left(\bigoplus_{k=1}^{K} \boldsymbol{\Omega}_{l l k}\right) \\
& =\bigoplus_{l=1}^{L}\left(\bigoplus_{k=1}^{K}\left(\varphi_{l l k} \xi_{l l k} a_{l l k} \mathbf{I}_{N_{t}}\right)\right) \\
& \stackrel{(a)}{=} \bigoplus_{l=1}^{L}\left(\bigoplus_{k=1}^{K}\left(\frac{\varphi_{l k k}^{\mathrm{twin}} \xi_{l l k} a_{l l k}}{\left(1+d_{l l k}\right)^{v} \mathbf{I}_{N_{t}}}\right)\right) \\
& \stackrel{\text { (b) }}{=} \operatorname{diag}\left\{\frac{\widetilde{\varphi}_{m}^{\mathrm{twin}} \tilde{\xi}_{m} \widetilde{a}_{m}}{\left(1+\widetilde{d}_{m}\right)^{v}}\right\}_{m=1}^{L K N_{t}},
\end{aligned}
$$

where for (a), $\varphi_{l l k}^{\mathrm{twin}}=\varphi_{l l k}^{\mathrm{tw}} \varphi_{l l k}^{\mathrm{in}}$ when $1 \leq k \leq K_{e}$ and $\varphi_{l l k}^{\mathrm{twin}}=1$ when $K_{e}<k \leq K$, the coefficients in (b) $\widetilde{\varphi}_{m}^{\text {twin }}$, $\widetilde{\xi}_{m}, \widetilde{a}_{m}$ represent path loss (outdoor path loss, I2O path loss), lognormal shadowing fading, and antenna radiation pattern, respectively. (b) is diagonal matrix of block diagonal matrix (a). These parameters can be determined by the formulas of (2), (3), and (7).

We hereafter investigate the sum rate performance of the 3D MIMO ZF receivers under BS cooperation. Under this consideration, we assume that BSs have perfect CSI which can be obtained by channel reciprocity in TDD and feedback in FDD. The processing of the ZF receiving is formulized as

$$
\mathrm{T}^{\dagger}=\left(\frac{P}{L K N_{t}}\right)^{-1 / 2}\left(\mathbf{G}^{H} \mathbf{G}\right)^{-1} \mathbf{G}^{H} .
$$

From (8) and (12), the instantaneous receive SNR at the $m$ th output is similar to [28]

$$
\gamma_{m}=\frac{\gamma[\boldsymbol{\Omega}]_{m m}}{L K N_{t}\left[\left(\mathbf{H}^{H} \mathbf{H}\right)^{-1}\right]_{m m}},
$$

where $\gamma=P / N_{0}$ is defined as the UT transmit power normalized by the receiver noise power. Hence, the achievable sum rate is achieved as

$$
R^{\mathrm{sum}} \triangleq \sum_{m=1}^{L K N_{t}} E\left[\log _{2}\left(1+\gamma_{m}\right)\right] .
$$


The expectation is taken over all channel realizations of $\mathbf{H}$, $\boldsymbol{\Omega}$ and the channel is assumed to be ergodic. It is worth mentioning that the sum rate $R^{\text {sum }}$ is contributed by two components of UTs: (i) cell-edge UTs and (ii) cell-center UTs.

Clearly, the main challenge in analytically evaluating (14) is to determine the closed-form expression for the PDF of SNR $\gamma_{m}$ in the case of Rayleigh lognormal (RLN) semicorrelated fading channel. To the best of authors' knowledge, the PDF of RLN is not in closed form and the approximation computations involve cumbersome functions. In order to bypass this problem, we exploit the accurate knowledge of the statistic of SNR $\gamma_{m}$ to derive a novel tractable lower bound on the sum rate of $3 \mathrm{D}$ MIMO ZF receivers, which enables us to pursue a statistical characterization of the massive MIMO systems.

The main interesting point of this paper is to optimize $R^{\text {sum }}$ with respect to some parameters, for example, the number of BS antennas, the number of UTs, the number of UT antennas, the location of the building, and orientation of antennas. To achieve this, we assume that the statistical information such as user location (coordinates) and channel statistics information are available at the BSs. This assumption is reasonable in an environment with low or moderate mobility, so long pilot sequence can be afforded and the UT coordinates can be easily obtained in the future systems, where the UTs are equipped with global positioning systems (GPS).

\section{Closed Form Bounds on the Sum Rate}

In the following, we derive a novel closed-form lower bound on the sum rate of 3D MIMO system under ZF receivers based on the result of [24]. For the convenience of exposition, the $3 \mathrm{D}$ MIMO correlation matrix $\mathbf{W} \triangleq \mathbf{H}^{H} \mathbf{H}$ will be extensively used in this paper. We begin the derivation with the following theorem which introduces a novel lower bound for RLN 3D MIMO channels for cell-edge UTs and cell-center UTs.

Theorem 1. The lower bound of the achievable sum rate performance of ZF receivers overcorrelated RLN 3D MIMO channels is given as follows:

$$
\begin{aligned}
& R_{L}^{\text {sum }}=\sum_{m=1}^{L K N_{t}} \log _{2}(1+\frac{\gamma}{L K N_{t}} \frac{\widetilde{\varphi}_{m^{\prime}}^{t w i n} \tilde{a}_{m^{\prime}}}{\left(1+\widetilde{d}_{m^{\prime}}\right)^{v}} \\
& \times \exp \left(\psi\left(L\left(N_{r}-K N_{t}\right)+1\right)+\frac{\mu_{m^{\prime}}}{\eta}\right. \\
&\left.\left.-\ln \left[\left(\bigoplus_{m^{\prime}=1}^{L K} \mathbf{R}_{m^{\prime}}\right)^{-1}\right]_{m m}\right)\right),
\end{aligned}
$$

where $m^{\prime}=\left\lceil m / N_{t}\right\rceil . \psi(\cdot)$ is Euler's digamma function [29, equation (8.360.1)].
Proof. Starting from (13) and (14), we obtain

$$
\begin{array}{r}
R^{\text {sum }}=\sum_{m=1}^{L K N_{t}} E\left[\log _{2}\left(1+\frac{\gamma}{L K N_{t}} \frac{[\mathbf{\Omega}]_{m m}}{\left[(\mathbf{W})^{-1}\right]_{m m}}\right)\right] \\
=\sum_{m=1}^{L K N_{t}} E\left[\operatorname { l o g } _ { 2 } \left(1+\frac{\gamma}{L K N_{t}}\right.\right. \\
\quad \times \exp \left(\ln \left([\mathbf{\Omega}]_{m m}\right)+\ln (\operatorname{det}(\mathbf{W}))\right. \\
\left.\left.\left.-\ln \left(\operatorname{det}\left(\mathbf{W}_{m m}\right)\right)\right)\right)\right],
\end{array}
$$

where from formula (16) to (17) we have used the equivalence transformation $x=\exp (\ln (x))$ and the following key matrix property [30]:

$$
\left[(\mathbf{W})^{-1}\right]_{m m}=\frac{\operatorname{det}\left(\mathbf{W}_{m m}\right)}{\operatorname{det}(\mathbf{W})}=\frac{\operatorname{det}\left(\mathbf{H}_{m}^{H} \mathbf{H}_{m}\right)}{\operatorname{det}\left(\mathbf{H}^{H} \mathbf{H}\right)},
$$

where $\mathbf{H}_{k}$ corresponds to $\mathbf{H}$ with the $k$ th column removed. By using Jensen's inequality, (17) is lower bounded by

$$
\begin{aligned}
R_{L}^{\text {sum }}=\sum_{m=1}^{L K N_{t}}\left[\log _{2}(1\right. & +\frac{\gamma}{L K N_{t}} \\
& \times \exp \left(E\left(\ln \left([\mathbf{\Omega}]_{m m}\right)\right)+E(\ln (\operatorname{det}(\mathbf{W})))\right. \\
& \left.\left.\left.-E\left(\ln \left(\operatorname{det}\left(\mathbf{W}_{m m}\right)\right)\right)\right)\right)\right]
\end{aligned}
$$

Exploiting the determinant property of square matrices $\operatorname{det}(\mathbf{A B})=\operatorname{det}(\mathbf{A}) \operatorname{det}(\mathbf{B})$, we can get

$$
\begin{aligned}
E\left[\log _{2}\left(\operatorname{det}\left(\mathbf{H}^{H} \mathbf{H}\right)\right)\right]= & E\left[\log _{2}\left(\operatorname{det}\left(\mathbf{V}^{H} \mathbf{V}\right)\right)\right] \\
& +E\left[\log _{2}(\operatorname{det}(\mathbf{R}))\right]
\end{aligned}
$$

and a similar manipulation for $E\left[\log _{2}\left(\operatorname{det}\left(\mathbf{H}_{m}^{H} \mathbf{H}_{m}\right)\right)\right]$.

For the $\mathbf{V}^{H} \mathbf{V}$, it is a central Wishart matrix $\mathbf{V}^{H} \mathbf{V} \sim$ $W_{L K N_{t}}\left(L N_{r}, \mathbf{I}\right)$ with $L N_{r} \geq L K N_{t}[30$, equation (2.12)]:

$$
E\left[\ln \left(\operatorname{det}\left(\mathbf{V}^{H} \mathbf{V}\right)\right)\right]=\sum_{m=0}^{L K N_{t}-1} \psi\left(L N_{r}-m\right) .
$$

For shadowing fading, we use the fundamental property of a lognormal RV $\xi_{m} \sim L N\left(\mu_{m} / \eta, \sigma_{m}^{2} / 2 \eta^{2}\right)[24]$ :

$$
E\left[\ln \xi_{m}\right]=\frac{\mu_{m}}{\eta}
$$

By substituting (11), (20), (21), and (22) into (19), we can obtain (15) after noting that

$$
\mathbf{H}_{m} \sim C N\left(\mathbf{0}_{L N_{r} \times L K N_{t}}, \mathbf{I}_{L N_{r}} \otimes \mathbf{I}_{L K N_{t}-1}\right) .
$$

Here $\otimes$ denotes the Kronecker product, and after some simple simplifications, we conclude the proof. 
Corollary 2. For the uncorrelated $R L N$ fading $\left(\mathbf{R}=\mathbf{I}_{L K N_{t}}\right)$, the sum rate of $3 D$ MIMO ZF receivers is bounded by

$$
\begin{aligned}
R_{L}^{\text {sum }}=N_{t} \sum_{m=1}^{L K} \log _{2}(1 & +\frac{\gamma}{L K N_{t}} \frac{\widetilde{\varphi}_{m}^{t w i n} \widetilde{a}_{m}}{\left(1+\widetilde{d}_{m}\right)} \\
& \left.\times \exp \left(\psi\left(L N_{r}-L K N_{t}+1\right)+\frac{\mu_{m}}{\eta}\right)\right) .
\end{aligned}
$$

Proof. By substituting $\mathbf{R}=\mathbf{I}_{L K N_{t}}$ into (15), we can easily conclude the proof.

It is easily seen that the sum rate monotonically grows with the mean of the lognormal shadowing, the antenna gain of both horizontal and vertical antenna radiation, and the number of BS antennas. However larger transceiver distance reduces the sum rate due to the increased path loss. Finally, we can observe that increasing the number of UTs or UT antennas do not always improve the system performance.

\section{Asymptotic and Optimal UTs Analysis of 3D MIMO Systems}

In this section, we investigate two issues: (i) the asymptotic sum rate for large transceiver antennas and (ii) the optimal number of UTs to achieve maximized sum rate.

4.1. Asymptotic Analysis. By deploying large antenna arrays on BS, we can save the transceiver powers both UTs and BSs when the number of antennas grows large, while maintaining a given desired quality of service (QoS). In the following, we derive the asymptotic achievable sum rates for $3 \mathrm{D}$ massive MIMO system.

(1) Fixed $\gamma, L, N_{t}, K$, and $N_{r} \rightarrow \infty$. Intuitively, when the number of BS antennas grows into infinity whilst keeping $\gamma, L$, $N_{t}$, and $K$ fixed, the 3D MIMO system captures more spatial diversity and array gains without bound.

Corollary 3. For 3D MIMO fading channels, the number of receive antennas tends to be unlimited with keeping a fixed $\gamma$, $L, N_{t}$, and $K$. The lower bound approaches

$$
\begin{aligned}
R_{L}^{\text {sum }} \underset{r}{N_{r}} \underset{\approx}{\approx} \sum_{m=1}^{L K N_{t}} \log _{2}\left(1+\frac{\gamma \widetilde{\varphi}_{m}^{t w i n} \tilde{a}_{m}}{\left(1+\widetilde{d}_{m}\right)^{v}}\left(\frac{N_{r}}{K N_{t}}-1\right)\right. \\
\times \exp \left(\frac{\mu_{m}}{\eta}\right. \\
\left.\left.-\ln \left[\left(\bigoplus_{m^{\prime}=1}^{L K} \mathbf{R}_{m^{\prime}}\right)^{-1}\right]_{m m}\right)\right)
\end{aligned}
$$

Proof. The proof follows by taking $N_{r}$ large in (15) with the asymptotic property of the digamma function [24]

$$
\psi(x) \approx \ln (x)+\frac{1}{x}+\mathrm{O}\left(\frac{1}{x^{2}}\right) \quad \text { if } x \longrightarrow \infty
$$

and substituting (26) into (15), we can obtain

$$
\begin{aligned}
R_{L}^{\text {sum }} \approx \sum_{m=1}^{L K N_{t}} \log _{2}\left(1+\frac{\gamma}{L K N_{t}} \frac{\widetilde{\varphi}_{m^{\prime}}^{\mathrm{twin}} \widetilde{a}_{m^{\prime}}}{\left(1+\widetilde{d}_{m^{\prime}}\right)}\right. & \\
\times \exp \left(\ln \left(L\left(N_{r}-K N\right)_{t}\right)\right. & \\
& +\frac{1}{L\left(N_{r}-K N_{t}\right)}+\frac{\mu_{m^{\prime}}}{\eta} \\
& \left.\left.-\ln \left[\left(\bigoplus_{m^{\prime}=1}^{L K} \mathbf{R}_{m^{\prime}}\right)^{-1}\right]_{m m}\right)\right) .
\end{aligned}
$$

For $N_{r} \rightarrow \infty$, we can further re-express (27) as follows:

$$
\begin{aligned}
R_{L}^{\mathrm{sum}}=\sum_{m=1}^{L K N_{t}} \log _{2} & \left(1+\frac{\gamma}{L K N_{t}} \frac{\widetilde{\varphi}_{m^{\prime}}^{\mathrm{twin}} \tilde{a}_{m^{\prime}}}{\left(1+\widetilde{d}_{m^{\prime}}\right)} L\left(N_{r}-K N_{t}\right)\right. \\
& \left.\times \exp \left(\frac{\mu_{m^{\prime}}}{\eta}-\ln \left[\left(\bigoplus_{m^{\prime}=1}^{L K} \mathbf{R}_{m^{\prime}}\right)^{-1}\right]_{m m}\right)\right) .
\end{aligned}
$$

After some algebraic simplifications, we can conclude the proof.

The above corollary reveals that, when the number of receive antennas grows into infinity, the effects of smallscale fading and noise disappear. Furthermore, we can see that the asymptotic sum rate grows logarithmically with $N_{r}$ without bound and decreases with transceiver distances and the correlation of UTs. This is consistent with [31].

(2) Fixed $\gamma, L, \kappa=N_{r} /\left(K N_{t}\right)$, and $N_{r} \rightarrow \infty$. This is an interesting asymptotic scenario that the number of BS antennas is large but may not be much greater than the number of UT antennas $K N_{t}$. Note that this scenario consists of two separate cases: (a) fixed $K$ and $N_{t} \rightarrow \infty$ and (b) fixed $N_{t}$ and $K \rightarrow \infty$. In case (a), it is a simple case that the bound scales linearly with the transmit antenna $N_{t}$. Besides, deploying a large number of antennas in the UTs is unrealistic. Therefore, we only take the case (b) into account. 
Corollary 4. For the 3D MIMO channels, as the numbers of $B S$ and UT antennas grow to infinity with a fixed ratio $\kappa=$ $N_{r} /\left(K N_{t}\right)>1$, the lower bound $R_{L}^{\text {sum }}$ converges to

$$
\begin{aligned}
R_{L} \stackrel{N_{r}, K \rightarrow \infty}{\approx} N_{t} \sum_{m=1}^{L K} \log _{2}\left(1+\frac{\gamma(\kappa-1) \widetilde{\varphi}_{m}^{t w i n} \tilde{a}_{m}}{\left(1+\widetilde{d}_{m}\right)^{v}}\right. \\
\times \exp \left(\frac{\mu_{m}}{\eta}\right. \\
\left.\left.\quad-\ln \left[\left(\bigoplus_{m^{\prime}=1}^{L K} \mathbf{R}_{m^{\prime}}\right)^{-1}\right]_{m m}\right)\right) .
\end{aligned}
$$

Proof. The proof follows, by substituting (26) into (15), that the lower bound $R_{L}^{\text {sum }}$ admits the following simplification:

$$
\begin{aligned}
& R_{L} \approx \sum_{m=1}^{L K N_{t}} \log _{2}\left(1+\frac{\gamma}{L K N_{t}} \frac{\widetilde{\varphi}_{m}^{\mathrm{twin}} \tilde{a}_{m}}{\left(1+\tilde{d}_{m}\right)^{v}}\right. \\
& \times \exp \left(\ln \left(L N_{r}-L K N_{t}\right)\right. \\
& \left.+\frac{\mu_{m}}{\eta}-\ln \left[\left(\bigoplus_{m^{\prime}=1}^{L K} \mathbf{R}_{m^{\prime}}\right)^{-1}\right]_{m m}\right) \\
& =N_{t} \sum_{m=1}^{L K} \log _{2}\left(1+\frac{\gamma L\left(N_{r}-K N_{t}\right)}{L K N_{t}\left(1+\widetilde{d}_{m}\right)^{v}}\right. \\
& \left.\times \exp \left(\frac{\mu_{m}}{\eta}-\ln \left[\left(\bigoplus_{m^{\prime}=1}^{L K} \mathbf{R}_{m^{\prime}}\right)^{-1}\right]_{m m}\right)\right) .
\end{aligned}
$$

Substituting $\kappa=N_{r} /\left(K N_{t}\right)$ into (30), we can further rewrite the

$$
\begin{aligned}
R_{L}^{\text {sum }}=N_{t} \sum_{m=1}^{L K} \log _{2}\left(1+\frac{\gamma(\kappa-1) \tilde{\varphi}_{m}^{\mathrm{twin}} \tilde{a}_{m}}{\left(1+\widetilde{d}_{m}\right)^{v}}\right. \\
\left.\quad \times \exp \left(\frac{\mu_{m}}{\eta}-\ln \left[\left(\bigoplus_{m^{\prime}=1}^{L K} \mathbf{R}_{m^{\prime}}\right)^{-1}\right]_{m m}\right)\right)
\end{aligned}
$$

and some simple simplifications; we can complete the proof.

Clearly, the asymptotic lower bound increases linearly with the number of UTs, $K$, and logarithmically with $\gamma$ and $\kappa$ while decreases with the distance $d$ and the correlation of UTs. The result above also indicates that the bound is achievable under the case $\kappa>1$. According to the law of large number, the bound can be further simplified which is an important topic for further work, though we do not pursue in this paper.

(3) Fixed $L, N_{t}, \gamma N_{r}$ and $N_{r} \rightarrow \infty$. Let $\gamma=E_{u} / N_{r}$, where $E_{u}$ is fixed. In this scenario, we also consider two separate cases: (a) fixed $K$ and (b) $K, N_{r} \rightarrow \infty$ and $\kappa=N_{r} /\left(K N_{t}\right)$. For case (a), we have the following corollary.

Corollary 5. For the 3D MIMO channels, as the number of receive antennas increases infinitely with $E_{u}, L, K$, and $N_{t}$ being fixed, the lower bound is bounded by

$$
\begin{aligned}
R_{L}^{\text {sum }}{ }_{r} \underset{\approx}{\approx} N_{t} \sum_{m=1}^{L K} \log _{2}\left(1+\frac{E_{u}}{K N_{t}} \frac{\widetilde{\varphi}_{m}^{t w i n} \tilde{a}_{m}}{\left(1+\tilde{d}_{m}\right)^{v}}\right. \\
\times \exp \left(\frac{\mu_{m}}{\eta}\right. \\
\left.\left.-\ln \left[\left(\bigoplus_{m^{\prime}=1}^{L K} \mathbf{R}_{m^{\prime}}\right)^{-1}\right]_{m m}\right)\right) .
\end{aligned}
$$

Proof. Substituting (26) and $\gamma=E_{u} / N_{r}$ into (15), we have the following expression:

$$
\begin{array}{r}
R_{L}^{\text {sum }} \underset{r}{N_{r}} N_{t} \sum_{m=1}^{L K} \log _{2}\left(1+\frac{E_{u}\left(N_{r}-K N_{t}\right)}{N_{r} K N_{t}} \frac{\widetilde{\varphi}_{m}^{\mathrm{twin}} \tilde{a}_{m}}{\left(1+\tilde{d}_{m}\right)^{v}}\right. \\
\times \exp \left(\frac{\mu_{m}}{\eta}\right. \\
\left.\left.-\ln \left[\left(\bigoplus_{m^{\prime}=1}^{L K} \mathbf{R}_{m^{\prime}}\right)^{-1}\right]\right)\right)
\end{array}
$$

For the case of $N_{r} \gg K N_{t}$, the lower bound (33) can be further simplified as

$$
\begin{aligned}
R_{L}^{\text {sum }}=N_{t} \sum_{m=1}^{L K} \log _{2} & \left(1+\frac{E_{u}}{K N_{t}} \frac{\widetilde{\varphi}_{m}^{\mathrm{twin}} \tilde{a}_{m}}{\left(1+\tilde{d}_{m}\right)^{v}}\right. \\
& \left.\times \exp \left(\frac{\mu_{m}}{\eta}-\ln \left[\left(\bigoplus_{m^{\prime}=1}^{L K} \mathbf{R}_{m^{\prime}}\right)^{-1}\right]_{m m}\right)\right) .
\end{aligned}
$$

We conclude the proof. 
Corollary 6. For the 3D MIMO channels, with the number of receive antennas growing to infinite with fixed $E_{u}, L, N_{t}$, and $\kappa=N_{r} /\left(K N_{t}\right)$, the lower bound is given by

$$
\begin{aligned}
R_{L}= & N_{t} \sum_{m=1}^{L K} \log _{2}\left(1+\frac{(\kappa-1) E_{u}}{\kappa K N_{t}} \frac{\widetilde{\varphi}_{m}^{t w i n} \widetilde{a}_{m}}{\left(1+\widetilde{d}_{m}\right)^{v}}\right) \\
& \times \exp \left(\frac{\mu_{m}}{\eta}-\ln \left[\left(\bigoplus_{m^{\prime}=1}^{L K} \mathbf{R}_{m^{\prime}}\right)^{-1}\right]_{m m}\right) .
\end{aligned}
$$

Proof. The proof is similar to Corollary 4 with $\kappa=N_{r} /\left(K N_{t}\right)$.

The Corollaries 5 and 6 reveal that, through using a very large antenna array at the receiver, the transmit power of each UT can be cut proportionally to $1 / N$ for maintaining a desired QoS. The similar result was originally proposed in [32]. Besides, we can see that when $\kappa$ tends to infinity, the two asymptotic results are identical with the results of Corollaries 3 and 4 . For Corollary 6, we can observe that the sum rate tends to a deterministic constant when $N_{r}$ grows large.

4.2. Optimal Number of UTs. In this section, the optimal number of UTs to maximize the system performance is investigated. From the former discussion, when the number of the BS antennas $N_{r} \rightarrow \infty$, the effect of Rayleigh fading can be perfectly canceled by $3 \mathrm{D}$ MIMO ZF receivers, but the effects of shadowing fading, path-loss, and antenna gain will remain. An optimal number of users $K^{\circ}$ exist because serving more users creates more interference which in turn reduces the sum rate of the users. At some point the sum rate will decrease because of the additional interference caused by extra UTs.

For simplicity, we consider a simple scenario that UTs are uniformly distributed on a circle of radius $r_{\text {building }}$, centered by the BSs, and that $\widetilde{\varphi}_{m}^{\mathrm{twin}}=\varphi, \widetilde{\mu}_{m}=\mu, \widetilde{\sigma}_{m}=\sigma, \widetilde{a}_{m}=a, \widetilde{d}_{m}=d$, and $m=1, \ldots, L K$. The optimal UT number is defined as the number of UTs which maximizes the approximate sum rate for a fixed received antenna number.

Consider the problem of finding the optimal number of UTs $K^{\circ}$ maximizing the approximated sum rate for a fixed receive antenna $N_{r}$

$$
\begin{aligned}
K^{\circ}=\underset{K}{\arg \max } L K N_{t} \log _{2}(1 & +\frac{\gamma \varphi e^{\mu / \eta} a}{L K N_{t}(1+d)^{v}} \\
& \left.\times \exp \left(\psi\left(L N_{r}-L K N_{t}+1\right)\right)\right),
\end{aligned}
$$

where $K^{\circ}$ denotes the optimal number of UTs.

To solve this problem, we begin with the following theorem which returns the approximated optimal solutions of (36).
Theorem 7. Under the condition of the assumptions above, the optimal number of UTs $K^{\circ}$ for maximizing the sum rate is given by

$$
K^{\circ}= \begin{cases}\frac{N_{r}}{e N_{t}} & \text { if } \Delta=1 \\ \frac{\Delta N_{r}}{N_{t}(\Delta-1)(1+(1 / W(x)))} & \text { else, }\end{cases}
$$

where $\Delta=\gamma \varphi a \exp (\mu / \eta) /(1+d)^{v}, x=(\Delta-1) / e$, and $W(x)$ is the Lambert $W$-function defined as $x=W(x) e^{W(x)}$.

Proof. For the assumptions above and $\Delta=1$, we can rewrite (36) as

$$
K^{\circ}=\underset{K}{\arg \max } L K N_{t} \log _{2}\left(1+\frac{\psi\left(L N_{r}-L K N_{t}\right)}{L K N_{t}}\right) .
$$

By using (26), the formula (38) can be expressed as

$$
\begin{aligned}
K^{\circ} & =\underset{K}{\arg \max } L K N_{t} \log _{2}\left(1+\frac{\left(N_{r}-K N_{t}\right)}{K N_{t}}\right) \\
& =\underset{K}{\arg \max } L K N_{t} \log _{2}\left(\frac{N_{r}}{K N_{t}}\right) .
\end{aligned}
$$

To maximize (39), we need to verify the concavity of the function with respect to $K$. The second derivative of (39) is firstly given by

$$
\frac{\partial^{2} R_{L}^{\text {sum }}}{\partial K^{2}}=-\frac{L N_{t}}{K}<0
$$

The inequality (40) reveals that the second derivative of the objective function is always negative. Therefore the objective function is concave with respect to the number of UTs $K$. Thus, we can obtain the optimal UTs by letting the first derivative be zero:

$$
\begin{gathered}
\frac{\partial R_{L}^{\text {sum }}}{\partial K}=\frac{L N_{t} \ln \left(N_{r} / K N_{t}\right)-L N_{t}}{\ln 2} \\
K^{\circ}=\frac{N_{r}}{e N_{t}}
\end{gathered}
$$

For $\Delta \neq 1$, after some simplifying, (36) can be further reexpressed as follows:

$$
K^{\circ}=\underset{K}{\arg \max } L N_{t} K \log _{2}\left(1+\frac{\Delta\left(N_{r}-K N_{t}\right)}{K N_{t}}\right) .
$$

Using the similar method to the case of $\Delta=1$, we need to verify the concavity of the function with respect to $K$. The second derivative of the (42) is given by

$$
\frac{\partial^{2} R_{U}}{\partial K^{2}}=\frac{-L \Delta^{2} N_{r}^{2} N_{t}}{K\left(K N_{t}(1-\Delta)+\Delta N_{r}\right)^{2}}<0 .
$$

The objective function is concave with respect to $K$ due to the negative of the second derivative of the objective function. 
Differentiating (42) along $K$ and letting the first derivative be zero, after some algebraic manipulations, we can obtain

$$
\ln \left(\frac{K N_{t}(1-\Delta)+\Delta N_{r}}{e K N_{t}}\right)=\frac{K N_{t}(\Delta-1)}{K N_{t}(1-\Delta)+\Delta N_{r}} .
$$

Denoting $W(K)=\left(K N_{t}(\Delta-1)\right) /\left(K N_{t}(\Delta-1)+\Delta N_{r}\right)$, and substituting $W(K)$ into (44), the expression of (44) can be rewritten as

$$
W(K) e^{W(K)}=K .
$$

Noticing the definition of $W(K)$ and solving for $K$, after some manipulations, we complete the proof for $\Delta \neq 1$. We can further conclude the whole proof.

As anticipated, the sum rate of 3D MIMO systems increases with the number of BS antennas. Also, we can conclude that the optimal number of UT does not always increase with the number of the transmit antennas. Therefore, we can achieve the maximum sum rate with the $\mathrm{ZF}$ receivers from the optimal solution of (37).

\section{Simulation Results}

In this section, we provide some numerical results to validate the accuracy of our analysis in a sectorized cell scenario. The multicell cellular system with $L$ cell cooperation is considered, as depicted in Figure 1. Each cell has a BS with a height of $h_{\mathrm{BS}}$ while the height of UT is $h_{\mathrm{UT}}$. The distance between the $\mathrm{BS}$ and the vertex of the cell is $R_{\text {cell }}$. The cell-edge UTs are located in a building with $F$ floors and the floor is simplified as a circle with radius $r_{\text {building. }}$. As for the cell-center UTs, they are uniformly distributed in the other area of the cell. The storey height is $h_{\text {floor }}$. All azimuth angles are given with respect to the center of the building. Tilting angles are given with respect to the ground and a positive value means downward tilting. The values of the system parameters used in the simulations are listed in Table 1. It is noteworthy that $N_{r}$ and $K$ are the number of BS antennas and UT in each sectorized cell.

We first investigate the tightness of the lower bound against the SNR in Figure 3 for different correlation coefficients $(\rho=0,0.5,0.8)$. In this paper, we consider exponential correlation model. The transmit correlation matrix is constructed as $R=\operatorname{diag}\left\{\operatorname{diag}\left\{R_{i k}\right\}_{k=1}^{K}\right\}_{i=1}^{L}$ where $R_{i k}$ is the correlation matrix among the antennas of the $k$ th UT in the $i$ th cell. The entries of the correlation matrix are modeled via the common exponential correlation model

$$
R_{i k p q}= \begin{cases}\rho^{|j-i|}, & i \leq j, \\ \left(\rho^{|j-i|}\right)^{*}, & i>j,\end{cases}
$$

where $|\rho|<1$ is the single correlation coefficient and $|\cdot|$ denotes the absolute value. It has been shown that the exponential model has been successfully used for many communication systems under rich scattering condition.

Clearly, the bound remains sufficiently tight across the entire SNR range for all correlation coefficients. At high

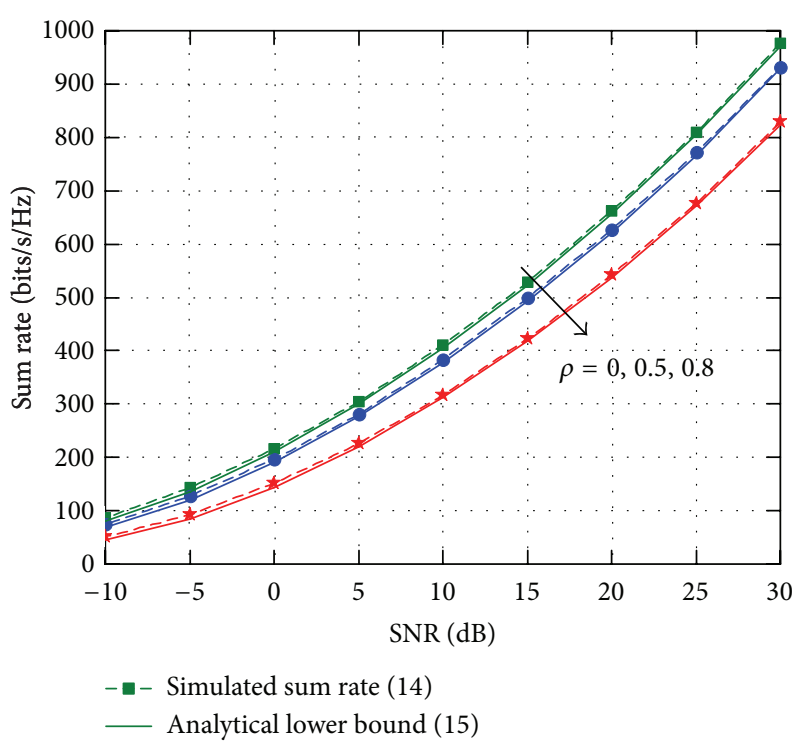

FigURE 3: Simulate sum rate and lower bound against the SNR $\gamma\left(N_{r}=40, N_{t}=2, K=18, \rho=0,0.5,0.8\right)$.

TABLE 1: Value of parameter in simulation.

\begin{tabular}{lcc}
\hline Parameter & Details & Value \\
\hline$L$ & Number of cells & 3 \\
$R_{\text {cell }}$ & Radius of sector & $50 \mathrm{~m}$ \\
$r_{\text {building }}$ & Radius of building & $10 \mathrm{~m}$ \\
$h_{\mathrm{BS}}$ & Height of BS & $30 \mathrm{~m}$ \\
$h_{\mathrm{UT}}$ & Height of UT & $1.5 \mathrm{~m}$ \\
$h_{\text {floor }}$ & Height of floor & $5 \mathrm{~m}$ \\
$\mu$ & Shadowing mean & $4 \mathrm{~dB}$ \\
$\sigma$ & Shadowing standard deviation & $4 \mathrm{~dB}$ \\
$v$ & Path-loss exponent & 4 \\
$F$ & Number of floors & 3 \\
$\varphi^{\text {tw }}$ & Wall penetration loss & $0.01(-20 \mathrm{~dB})$ \\
$\varphi^{\text {in }}$ & Inside loss & $0.5 d_{2 \mathrm{D}-\text { in }}$ \\
$A_{m}$ & Azimuth front-to-back ratio & $30 \mathrm{~dB}$ \\
$\phi_{3 \mathrm{~dB}}$ & Horizontal HPBW & $70^{\circ}$ \\
$\theta_{3 \mathrm{~dB}}$ & Vertical HPBW & $7^{\circ}$ \\
SLA & Side lobe attenuation & $30 \mathrm{~dB}$ \\
$\phi^{\text {orn }}$ & azimuth angles & $0^{\circ}, 120^{\circ},-120^{\circ}$ \\
$R_{e / c}$ & UT edge-cell to center-cell ratio & $1 / 3$ \\
$\beta^{m}$ & Mechanical tilt angle & $0^{\circ}$ \\
${ }^{*} d_{2 \mathrm{D}-\text { in }}$ denotes the distance from the wall to the indoor UT, which is given \\
in [23].
\end{tabular}

SNRs, the sum rate and the bounds increase approximately linearly with the SNRs. Moreover, the effect of correlation on the sum rate performance can be neglected at low SNR. From Figure 3, we also observe that when the correlation between antenna elements of the same UT increases, the sum rate decreases significantly.

The lower bound versus the BS tilt angle is shown in Figure 4 for three different correlation coefficients $\rho=0,0.5$, and 0.8 . It is shown that the sum rate increases with tilt 


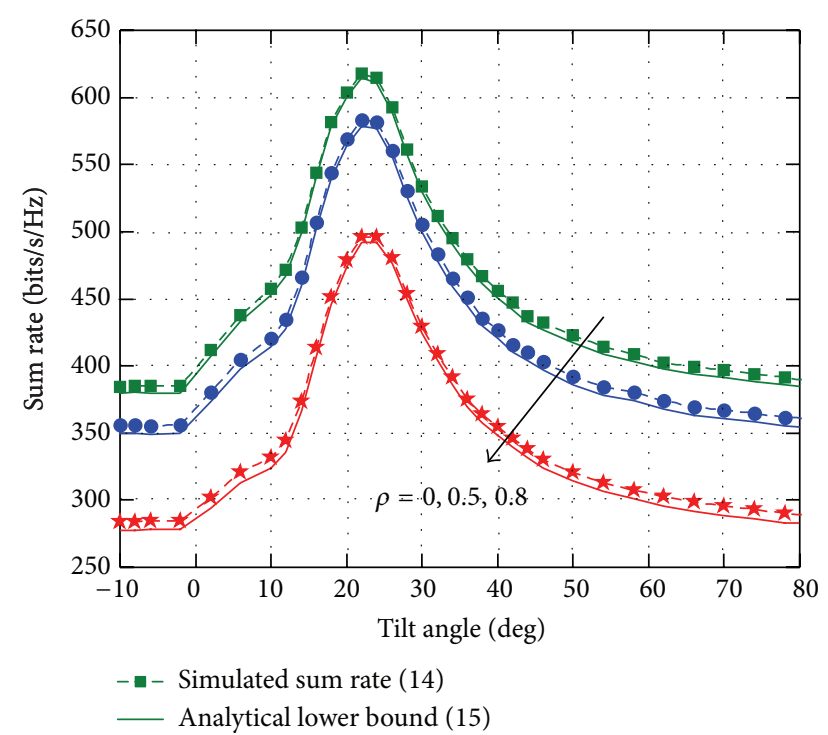

FIGURE 4: Simulate sum rate and lower bound versus tilt angle ( $\gamma=$ $\left.20 \mathrm{~dB}, N_{r}=50, N_{t}=2, K=18, \rho=0,0.5,0.8\right)$.

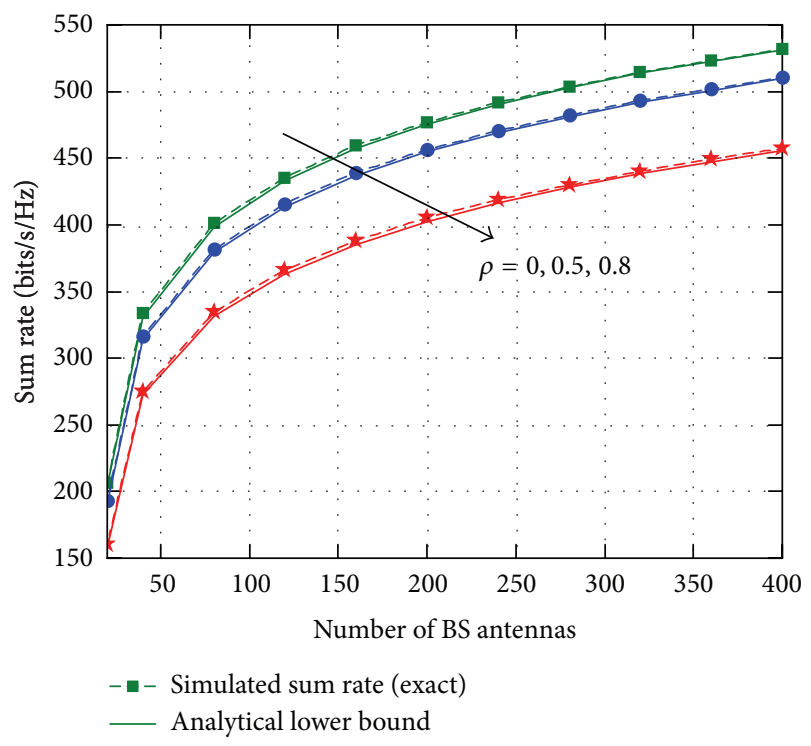

FIGURE 5: Analytical sum rate and lower bound versus the number of BS antennas ( $\left.\gamma=10 \mathrm{~dB}, N_{t}=2, K=9, \rho=0,0.5,0.8\right)$.

angle before the optimal angle (about $21^{\circ}$ ) and then decrease with the further increase. Besides, we can observe that, even for a small number of the receive antenna elements, the lower bound is sufficiently tight. The effects of correlation are also shown in Figure 4. As expected, when the correlation coefficient grows, the sum rate and the lower bound decrease.

In the following, the sum rate performance of the $3 \mathrm{D}$ massive MIMO system against the number of receive antennas for fixed $\gamma=10$ and $\gamma=10 / N_{r}$ are given in Figures 5 and 6 . As expected, with $\gamma=10 \mathrm{~dB}$, the sum rate and lower bound scale logarithmically with the number of receive antennas $N_{r}$ without bound for all correlation coefficients. However for $\gamma=10 / N_{r}$, the sum rate converges to a constant value when

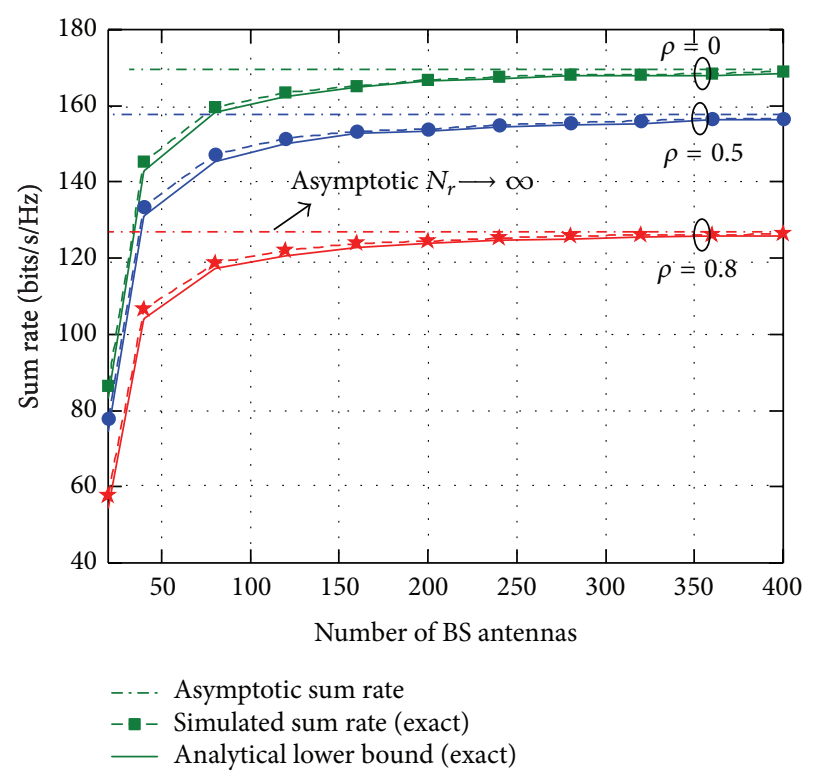

FIgURE 6: Analytical sum rate and lower bound versus the number of BS antennas $\left(\gamma=10 / N_{r} \mathrm{~dB}, N_{t}=2, K=9, \rho=0,0.5\right.$, and 0.8$)$.

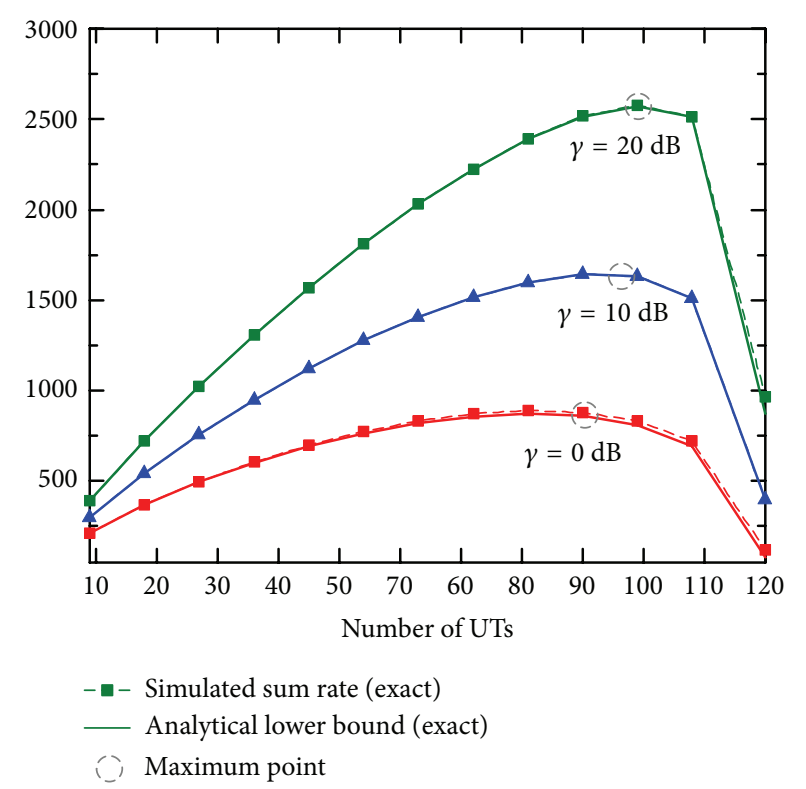

FIGURE 7: Analytical sum rate and lower bound versus the number of UTs $\left(\gamma=0,10,20 \mathrm{~dB}, N_{t}=1, N_{r}=120\right)$.

the number of receive antennas grows large. This is consistent with [31].

Finally, the optimal number of UTs $K^{\circ}$ is investigated. The sum rate in Figure 7 is a concave function as proved in (40) and (43). Thus there exists an optimal point of UT number $K$ that maximizes the sum rate. Figure 7 compares the optimal number of UT $K^{\circ}$ obtained in (37) and the $K$ by Monte Carlo simulation such that the sum rate is maximized.

From Figure 7, we observe that the optimal number of the UT $K^{\circ}$ obtained in (37) and $K, K \in\left\{1, \ldots, N_{r}\right\}$ obtained by Monte Carlo simulation are matched very well. In addition, it 
implies that the sum rate decreases instead of increasing with $K$ if the transmit power at the BS is not sufficiently large after serving a certain number (optimal number) of the UTs. The similar conclusion appears in [8]. Therefore, the study of the optimal UT number is of great practical significance.

\section{Conclusion}

This paper investigates the sum rate of the $3 \mathrm{D}$ massive MIMO system over BS cooperation and BS antenna tilt angle. More importantly, we derived a novel and simple closed form for the lower bound of the sum rate. Based on the lower bound, we analyzed in detail the promising technology of the massive MIMO systems. In parallel, we also investigate the impacts of large-scale (log-normal shadowing fading, 3D antenna gain, distance path-loss, and $\mathrm{I} 2 \mathrm{O}$ propagation loss) and small-scale fading, as well as the spatial correlation at the transmit side considering the cell-edge UTs and cell-center UTs. Besides, an expression is derived to obtain the approximate optimal number of UTs that maximizes the sum rate. This result has an important significance for the optimum UT configuration in practice. Although this model is simple, it can lead to easily following the derivations and quite insightful results on the benefits of MU-MIMO in a 3D MIMO and massive MIMO situations.

\section{Conflict of Interests}

The authors declare that there is no conflict of interests regarding the publication of this paper.

\section{Acknowledgments}

This work was supported by the National 863 Project (no. 2014AA01A705), the Creative Research Groups of China (61121001), and National Science and Technology Major Project (no. 2013ZX03003009).

\section{References}

[1] G. J. Foschini and M. J. Gans, "On limits of wireless communications in a fading environment when using multiple antennas," Wireless Personal Communications, vol. 6, no. 3, pp. 311-335, 1998.

[2] E. Telatar, "Capacity of multi-antenna Gaussian channels," European Transactions on Telecommunications, vol. 10, no. 6, pp. 585-595, 1999.

[3] F. Rusek, D. Persson, B. K. Lau et al., "Scaling up MIMO: opportunities and challenges with very large arrays," IEEE Signal Processing Magazine, vol. 30, no. 1, pp. 40-60, 2013.

[4] R. Couillet and M. Debbah, "Signal processing in large systems: a new paradigm," IEEE Signal Processing Magazine, vol. 30, no. 1, pp. 24-39, 2013.

[5] F. Boccardi, R. W. Heath, A. Lozano, T. L. Marzetta, and P. Popovski, "Five disruptive technology directions for 5G," IEEE Communications Magazine, vol. 52, no. 2, pp. 74-80, 2014.

[6] T. L. Marzetta, "Noncooperative cellular wireless with unlimited numbers of base station antennas," IEEE Transactions on Wireless Communications, vol. 9, no. 11, pp. 3590-3600, 2010.
[7] N. Gresset, H. Halbauer, J. Koppenborg, W. Zirwas, and H. Khanfir, "Interference-avoidance techniques: improving ubiquitous user experience," IEEE Vehicular Technology Magazine, vol. 7, no. 4, pp. 37-45, 2012.

[8] X. W. Li, L. H. Li, and L. Xie, "Achievable sum rate analysis of ZF receivers in 3D MIMO systems," KSII Transactions on Internet and Information Systems, vol. 8, no. 4, pp. 1368-1389, 2014.

[9] Y. Li, X. Ji, and D. Liang, "Dynamic beamforming for threedimensional MIMO technique in LTE-advanced networks," International Journal of Antennas and Propagation, vol. 2013, Article ID 764507, 8 pages, 2013.

[10] W. D. Zhang and Y. Wang, "Interference coordination with vertical beamforming in 3D MIMO-OFDMA networks," IEEE Communications Letters, vol. 18, no. 1, pp. 34-37, 2013.

[11] A. D. Wyner, "Shannon-theoretic approach to a Gaussian cellular multiple-access channel," IEEE Transactions on Information Theory, vol. 40, no. 6, pp. 1713-1727, 1994.

[12] D. Gesbert, S. Hanly, H. Huang, S. Shamai Shitz, O. Simeone, and W. Yu, "Multi-cell MIMO cooperative networks: a new look at interference," IEEE Journal on Selected Areas in Communications, vol. 28, no. 9, pp. 1380-1408, 2010.

[13] A. Lozano, R. W. Heath Jr., and J. G. Andrews, "Fundamental limits of cooperation," IEEE Transactions on Information Theory, vol. 59, no. 9, pp. 5213-5226, 2013.

[14] H. Dai and H. V. Poor, "Asymptotic spectral efficiency of multicell MIMO systems with frequency-flat fading," IEEE Transactions on Signal Processing, vol. 51, no. 11, pp. 2976-2988, 2003.

[15] N. Seifi, M. Coldrey, M. Matthaiou, and M. Viberg, "Impact of base station antenna tilt on the performance of network MIMO systems," in Proceeding of the 75th IEEE Vehicular Technology Conference (VTC Spring '12), pp. 1-5, Yokohama, Japan, June 2012.

[16] X. Lu, A. Tolli, O. Piirainen, M. Juntti, and W. Li, "Comparison of antenna arrays in a 3-D multiuser multicell network," in Proceedings of the IEEE International Conference on Communications (ICC '11), May 2011.

[17] O. Somekh and S. Shamai, "Shannon-theoretic approach to a Gaussian cellular multiple-access channel with fading," IEEE Transactions on Information Theory, vol. 46, no. 4, pp. 1401-1425, 2000.

[18] D. Aktas, M. N. Bacha, and J. S. Evans, "Scaling results on the sum capacity of cellular networks with MIMO links," IEEE Transactions on Information Theory, vol. 52, no. 7, pp. 32643274, 2006.

[19] S. Chatzinotas, M. A. Imran, and R. Hoshyar, "On the multicell processing capacity of the cellular MIMO uplink channel in correlated rayleigh fading environment," IEEE Transactions on Wireless Communications, vol. 8, no. 7, pp. 3704-3715, 2009.

[20] A. Muller, J. Hoydis, R. Couillet, and M. Cdebbah, “Optimal 3D cell planning: a random matrix approach," in Proceedings of the IEEE Global Communications Conference (GLOBECOM '12), pp. 4512-4517, Anaheim, Cali, USA, December 2012.

[21] K. Zheng, S. L. Ou, and X. F. Yin, "Massive MIMO channels: a survey," International Journal of Antennas and Propagation, vol. 2014, Article ID 848071, 10 pages, 2014.

[22] S. B. Wu, C. X. Wang, and H. Aggoune, "Non-stationary wideband channel models for massive MIMO systems," in Proceedings of the 2nd Symposium on Wireless Sensor and Cellular Networks (WSCN'13), Jeddah, Saudi Arabia, December 2013. 
[23] 3GPP TR 36.873 V1.0.0, “3D Channel Model for LTE”, September 2013.

[24] M. Matthaiou, C. Zhong, M. R. McKay, and T. Ratnarajah, "Sum rate analysis of ZF receivers in distributed MIMO systems," IEEE Journal on Selected Areas in Communications, vol. 31, no. 2, pp. 180-191, 2013.

[25] M. K. Simon and M. Alouini, Digital Communication over Fading Channel, John Wiley \& Sons, New York, NY, USA, 2004.

[26] H. Dai, H. Zhang, and Q. Zhou, "Some analysis in distributed MIMO systems," Journal of Communications, vol. 2, no. 3, pp. 43-50, 2007.

[27] T. G. Vasiliadis, A. G. Dimitriou, and G. D. Sergiadis, "A novel technique for the approximation of 3-D antenna radiation patterns," IEEE Transactions on Antennas and Propagation, vol. 53, no. 7, pp. 2212-2219, 2005.

[28] D. A. Gore, R. W. Heath Jr., and A. J. Paulraj, "Transmit selection in spatial multiplexing systems," IEEE Communications Letters, vol. 6, no. 11, pp. 491-493, 2002.

[29] I. S. Gradshteyn and I. M. Ryzhik, Table of Integrals, Series, and Products, Academic Press, New York, NY, USA, 7th edition, 2007.

[30] A. M. Tulino and S. Verdu, Random Matrix Theory and Wireless Communications, Now Publishers, Delft, The Netherlands, 2004.

[31] H. Q. Ngo, M. Matthaiou, T. D. Duong, and E. G. Larsson, "Uplink performance analysis of multicell MU-SIMO systems with ZF receivers," IEEE Transactions on Vehicular Technology, vol. 62, no. 9, pp. 4471-4483, 2013.

[32] H. Q. Ngo, E. G. Larsson, and T. L. Marzetta, "Energy and spectral efficiency of very large multiuser MIMO systems," IEEE Transactions on Communications, vol. 61, no. 4, pp. 1436-1449, 2013. 

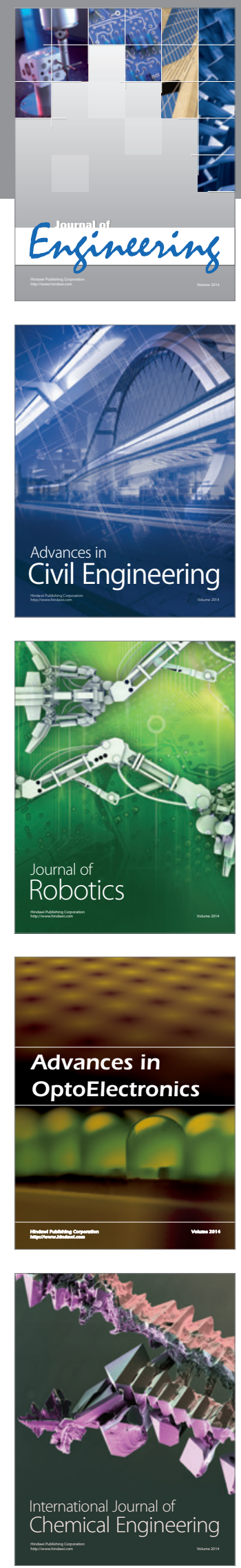

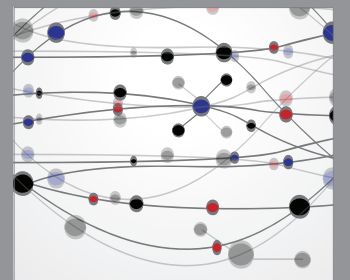

The Scientific World Journal
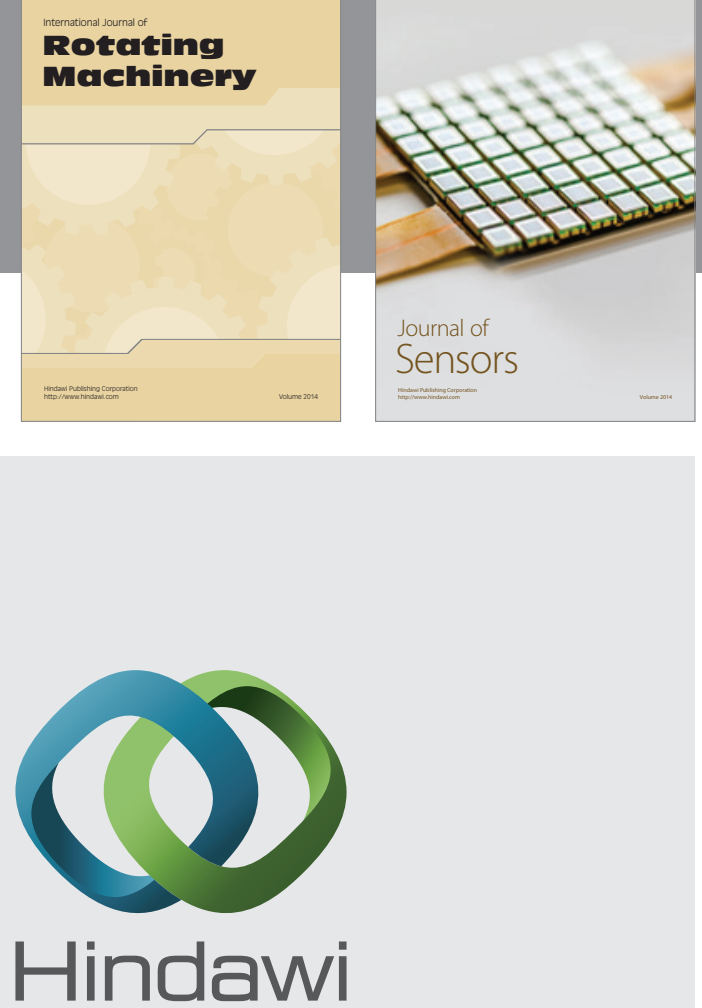

Submit your manuscripts at http://www.hindawi.com
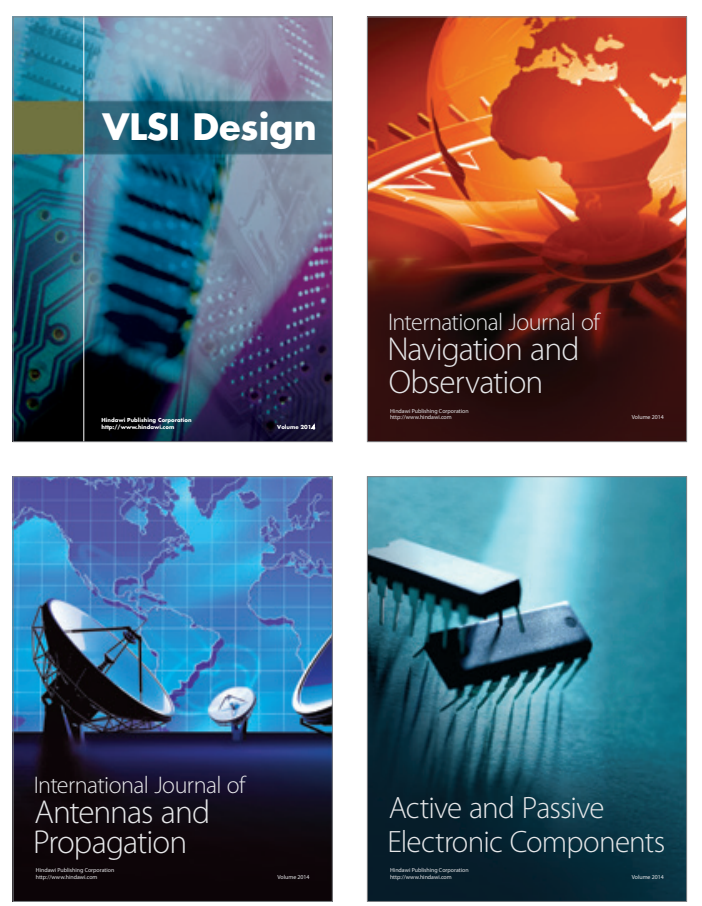
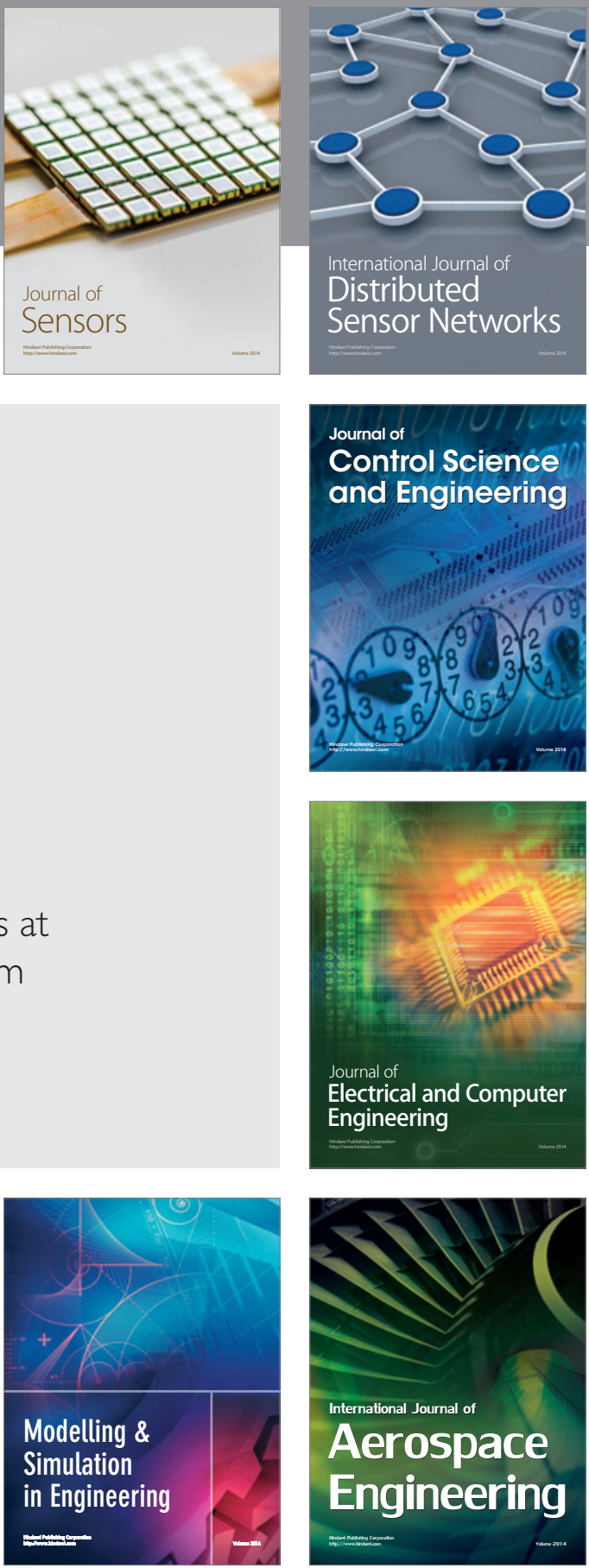

Journal of

Control Science

and Engineering
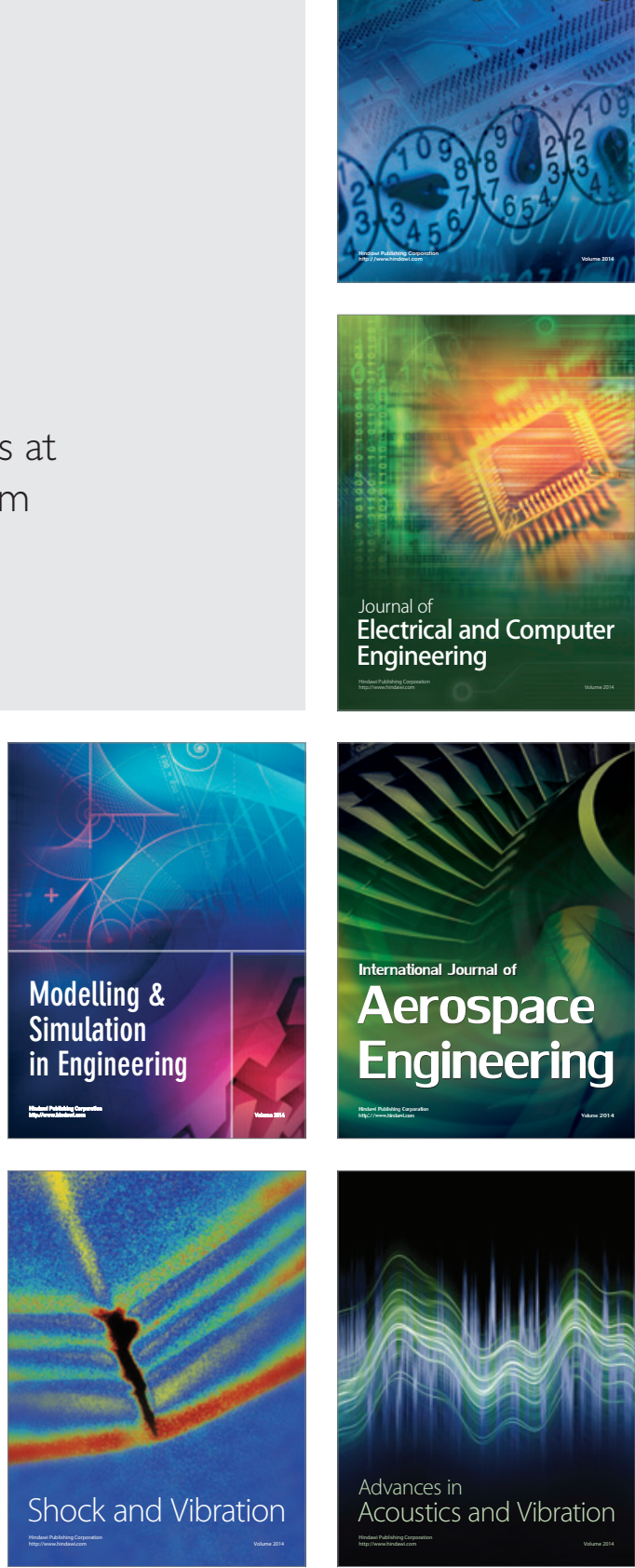